\title{
Evaluación del tratamiento de consolidación de dolomías mediante nanopartículas de hidróxido de calcio en condiciones de alta humedad relativa
}

\author{
L.S. GOMEZ-VILLALBA*, P. LÓPEZ-ARCE*, A. ZORNOZA*, M. ÁLVAREZ DE BUERGO*, R. FORT* \\ Instituto de Geociencias (CSIC-UCM) C.P. 28040, Madrid España
}

\begin{abstract}
En este artículo se presentan los resultados del tratamiento aplicado a muestras de piedra dolomía mediante una solución coloidal isopropílica basada en nanopartículas de hidróxido de calcio en una concentración de 2,0g/l. El proceso de consolidación de la piedra ha sido monitoreado antes y después de su aplicación a los 28 días de estar expuesta a una atmósfera del $75 \%$ de humedad relativa. Se ha realizado un estudio morfológico y estructural del consolidante confirmándose el proceso de carbonatación. Los resultados de difracción de rayos X, microscopía electrónica (TEM y ESEM) y difracción de electrones realizados al producto consolidante han confirmado la transformación de la fase portlandita a los polimorfos de carbonato de calcio de tipo calcita, aragonito y vaterita. Los ensayos petrofísicos realizados a la piedra antes y después de su aplicación, han demostrado que se produce una mejora de las propiedades físicas e hídricas indicado por el aumento tanto en la velocidad de propagación de ultrasonidos como en la densidad del material y una disminución de su coeficiente capilar, así como de la porosidad abierta sin producirse modificaciones significativas en el color y el brillo. La aplicación del tratamiento de consolidación propuesto, con este tipo de soluciones coloidales de nanopartículas, es un método natural y compatible con las características petrológicas del sustrato, sin generar daños secundarios sobre la piedra, resultando un método eficaz para la mejora de la durabilidad de rocas carbonáticas.
\end{abstract}

Palabras clave: Consolidación, piedra, polimorfos de carbonato de calcio, nanopartículas de hidróxido cálcico, humedad relativa.

Evaluation of a consolidation treatment in dolostones by mean of calcium hydroxide nanoparticles in high relative humidity conditions

In this article, the results of a treatment applied to dolomitic stones using an isopropyl colloidal solution based on calcium hydroxide nanoparticles with a concentration of $2.0 \mathrm{~g} / 1$ are presented. The consolidation process in the stone has been checked before and after 28 days of exposure to 75\% relative humidity. Morphologic and structural studies of the consolidating product confirmed the carbonation process. $X$ ray diffraction, electron microscopy (TEM and ESEM), and electron diffraction carried out on the consolidating product have confirmed the transformation of portlandite phase to calcium carbonate polymorphs, calcite, aragonite and vaterite. Petrophysical tests performed on the stone before and after the application of the product have shown the improvement in the physical and hydrical properties due to the increase in the ultrasound velocity and density of the material, and a decrease in the capillarity coefficient and open porosity without significant changes in colour and brightness. The application of the consolidating product in the proposed experimental conditions is a natural method, compatible with the petrological characteristics of the substrate, without secondary damages on the stone, being an effective method to improve the durability of carbonate stones.

Keywords: Consolidation, stone, calcium carbonate polymorphs, calcium hydroxide nanoparticles, relative humidity.

\section{INTRODUCCIÓN}

Dentro de los materiales de construcción, la dolomía ha sido uno de los materiales pétreos más ampliamente utilizados en patrimonio arquitectónico y escultórico. Este tipo de roca carbonatada está compuesta predominantemente por dolomita (carbonato de calcio y magnesio) aunque se encuentra asociada en cantidades variables a carbonatos de calcio (calcita). Además de ser empleada como roca ornamental, también ha sido utilizada para áridos de machaqueo, morteros y en la fabricación de cerámicas y pinturas.

Como todos los materiales que constituyen el patrimonio cultural histórico y construido, la dolomía está sometida a diferentes procesos de deterioro, bien por las características intrínsecas de los mismos- principalmente mineralogía y textura-, bien por agentes externos. Entre estos últimos, cabe destacar la acción del agua, la presencia de sales y el causado por el uso inapropiado de ciertos tratamientos reparadores de conservación [1] [2], sin olvidar el deterioro acelerado que pueden generar las condiciones medioambientales agresivas a las que se ven sometidos en ocasiones los materiales [3], [4] .

El deterioro resultante puede generar una pérdida de la cohesión interna de los constituyentes de los materiales, con pérdida física de material y del valor histórico y cultural de los elementos patrimoniales. Para remediar este hecho, se utilizan productos consolidantes, cuya misión es la de 
restablecer la cohesión perdida. Los productos que se han venido utilizando hasta la fecha pueden clasificarse como de naturaleza orgánica (bien naturales o sintéticos), inorgánica o mixtos (organominerales). La eficacia de dichos productos depende de las características del substrato sobre los que se aplique, de las propiedades del producto en sí, de la compatibilidad producto-substrato, y de las técnicas, métodos y condiciones de aplicación, entre otras muchas variables.

Respecto a los productos orgánicos y organominerales (también conocidos como organosilícicos), pueden resultar eficaces a corto y medio plazo. El principal problema que presentan es su durabilidad en el tiempo [5], ya que al poseer una naturaleza distinta a la del substrato mineral, sus propiedades son distintas y su comportamiento en el tiempo también diferente, es decir, evolucionan de forma desigual. Es conocido el hecho de la necesidad de reaplicación de este tipo de productos para conseguir una efectividad y durabilidad a largo plazo.

Por todo ello parece razonable emplear productos que sean más idóneos y compatibles con la naturaleza mineralógica del substrato pétreo deteriorado que se quiere consolidar. Así, uno de los consolidantes inorgánicos más conocidos desde tiempos históricos es el hidróxido cálcico [6] [7]. El método "limewater/ limewash putty method", se basa en el hecho de que cuando el hidróxido cálcico se expone al $\mathrm{CO}_{2}$ atmosférico en condiciones de humedad, se produce su carbonatación, reaccionando y transformándose en carbonato cálcico. Sin embargo, este método ha resultado en muchos casos insatisfactorio por la escasa penetración de la disolución en el interior del material pétreo y su imposibilidad por tanto para conseguir una consolidación completa de la parte deteriorada del material [8], [9].

El desarrollo en los últimos años de la nanociencia y la nanotecnología ha abierto la posibilidad a diferentes áreas científicas. Esta nueva ciencia posibilita nuevas aplicaciones de los materiales que anteriormente eran inviables, ya que a escala nanométrica el comportamiento de los materiales se ve modificado como resultado de la reducción del tamaño de partícula. La nanotecnología aporta a la ciencia de la conservación del patrimonio cultural nuevos productos que mejoran las propiedades de protección y consolidación de los materiales, con ventajas muy superiores a otros tratamientos tradicionales.

Es por ello que parece que la aplicación de nanopartículas de hidróxido cálcico dispersas en diferentes tipos de alcoholes para la consolidación de materiales carbonáticos puede llegar a solventar este problema [10], [11].

Un nanomaterial se define como aquel material que posee unas características estructurales donde al menos una de sus dimensiones está en el intervalo de 1-100 nanómetros. Una partícula de tamaño nanométrico proporciona un área específica superficial mayor para la interacción molecular y por tanto, incrementa su velocidad de reacción.

En los últimos años, a la par que ha avanzado la nanotecnología, las casas comerciales ofrecen productos para ser aplicados en diferentes campos de aplicación. Dentro de ellos existen actualmente en el mercado "nanomateriales" para ser aplicados como consolidantes en piedra, pinturas murales, materiales cerámicos, etc. Sin embargo, la estabilidad de dichos consolidantes y su eficacia están aún en fase de evaluación [12].

La utilización de nanopartículas de $\mathrm{Ca}(\mathrm{OH})_{2}$ para la consolidación de materiales carbonatados viene marcado por una serie de mejoras en sus propiedades para garantizar el éxito del proceso como son la morfología y el tamaño de los cristales, la tasa y el efecto de la carbonatación en el crecimiento cristalino y en el grado de cristalinidad, el control de la profundidad de penetración, la concentración de la solución, aditivos y disolventes, y las cuestiones de solubilidad de los productos [13].

En este trabajo se analizan las características de un consolidante comercial a base de nanopartículas de hidróxido cálcico, y se valora la eficacia de su aplicación a un material carbonático en función del tiempo y de la humedad relativa, por considerar que esta última variable juega un importante papel en el proceso de carbonatación del hidróxido cálcico.

A diferencia de los trabajos realizados anteriormente [10,11], el presente estudio está enfocado a evaluar los resultados de la aplicación de un producto comercial diferente, aunque igualmente basado en nanopartículas de hidróxido de calcio para aplicaciones como consolidante. En este caso, el consolidante utilizado ha sido obtenido por el mismo método de síntesis coloidal, aunque con diferentes condiciones experimentales [14]. Con el fin de evaluar el comportamiento de este producto se ha variado la concentración de la solución precursora utilizando el mismo disolvente y se ha modificado la aplicación del mismo sobre el material pétreo, tal y como se describe a continuación.

\section{MATERIALES Y MÉTODOS}

\subsection{Preparación de la solución precursora y proceso de aplicación del consolidante}

El producto utilizado como consolidante está basado en una solución coloidal comercial de hidróxido de calcio (CaLoSiL®) [14] disuelta en alcohol isopropílico con una concentración inicial de $20 \mathrm{~g} / 1$ a partir de la cual se obtuvo una suspensión isopropílica de $2,0 \mathrm{~g} / \mathrm{l}$. Dicha solución precursora fue incorporada en una cámara climática sin flujo extra de $\mathrm{CO}_{2^{\prime}} \sin$ aditivos, a una temperatura constante de $20^{\circ} \mathrm{C}$. La cámara climática fue adecuada a una humedad relativa del $75 \%$ mediante la aplicación de una solución salina supersaturada en $\mathrm{NaCl}$.

La piedra utilizada para evaluar el efecto del consolidante proviene de una dolomía de edad cretácica de la región de Redueña (Norte de Madrid-España), material muy utilizado en la construcción de edificios históricos españoles [15]. Esta dolomía está caracterizada por presentar una alta porosidad y consistencia deleznable, lo que la hace susceptible a agentes de deterioro por pérdida de cohesión del material.

Las muestras provienen de una antigua cantera de dónde antiguamente se extraía este tipo de variedad pétrea. Para su análisis se cortaron probetas de tamaño $2.5 \times 2.5 \times 1.5 \mathrm{~cm}$.

Para el proceso de consolidación con la solución coloidal, las probetas fueron depositadas en un vaso de precipitados de vidrio al cual se le añadió previamente $30 \mathrm{ml}$ aprox. de producto hasta cubrir $3 \mathrm{~mm}$ la base de la probetas. Éste se cubrió con parafilm, para evitar la evaporación del producto, y se introdujo a continuación en una bañera de ultrasonidos durante $2 \mathrm{~h}$, para permitir la penetración del producto en el sustrato pétreo por capilaridad ayudado por la dispersión inducida mediante ultrasonidos. Finalmente, las probetas se introdujeron en la cámara climática a $20^{\circ} \mathrm{C}$ de temperatura (T) y $75 \%$ de humedad relativa (HR), ya que en investigaciones previas se ha podido comprobar la mayor efectividad de este tipo de producto como tratamiento consolidante bajo estas condiciones [10]. La caracterización petrofísica de las probetas de piedra se realizó antes y después de 28 días de consolidación dentro de la cámara climática. 


\subsection{Técnicas de caracterización}

\subsubsection{CARACTERIZACIÓN DEL CONSOLIDANTE}

La caracterización morfológica y química de la solución coloidal, a 14 y 28 días de exposición, fue realizada mediante las técnicas de microscopía electrónica de barrido (ESEM) y microscopía electrónica de transmisión (TEM). Se utilizó el microscopio de barrido FEI Quanta 200 en modo de operación ambiental (ESEM) con detector de energía dispersiva de rayos $X$ (EDX) de análisis integrado OXFORD INSTRUMENTS ANALYTICAL-INCA y el microscopio de transmisión Jeol JEM 2000 a $200 \mathrm{KV}$ con detector de energía dispersiva de rayos $X$ Link, equipado con detector de difracción de electrones en modo selección de área (saed). El estudio microestructural y la identificación de fases cristalinas se realizó mediante las técnicas de difracción de rayos $\mathrm{X}$ y difracción de electrones. Se utilizó un difractómetro X`Pert Pro MPD Panalytical $X^{\wedge}$ Celerator, en un rango de $2 \theta$ de $5-80^{\circ}$, un tamaño de paso de $0,033^{\circ}$ y 200,025 segundos por paso en modo continuo.

\subsubsection{CARACTERIZACIÓN MINERALÓGICA Y TEXTURAL DE LA PIEDRA ANTES Y DESPUÉS DEL TRATAMIENTO}

La caracterización de la piedra antes de la aplicación del consolidante se realizó mediante las técnicas de microscopía óptica de luz polarizada transmitida y microscopía electrónica de barrido ambiental. Para el estudio petrográfico se elaboraron previamente láminas delgadas del material con una superficie de $3 \mathrm{~cm} \times 2 \mathrm{~cm}$ y un espesor de 30 micrómetros. Por medio del análisis petrográfico se determinaron las características texturales, la composición mineralógica, el tamaño de los cristales y el grado de alteración de los mismos utilizando un microscopio petrográfico de marca OLYMPUS BX51 con cámara digital acoplada.

La piedra antes y después de la aplicación de la solución coloidal fue analizada en el mismo microscopio electrónico de barrido en modo de operación ambiental (ESEM). Mediante este estudio se identificaron los minerales presentes, su morfología, grado de fracturación y tamaño de los poros en la muestra antes de la aplicación del consolidante. La misma superficie pétrea, una vez expuesta a la solución ( 28 días al 75\% HR), fue analizada identificando las variaciones como resultado de la aplicación del producto, determinando las fases mineralógicas formadas sobre el sustrato, alteración de la superficie y su efecto sobre la porosidad intra e intergranular. Estas mismas muestras fueron estudiadas mediante difracción de rayos $\mathrm{X}$ en las mismas condiciones que se realizó el análisis del producto consolidante.

El programa Digital Micrograph ${ }^{\mathrm{TM}}$ Gatan Inc. fue empleado para la interpretación de las imágenes obtenidas por ESEM, TEM y de los patrones de difracción de electrones.

\subsubsection{CARACTERIZACIÓN PETROFÍSICA}

Para evaluar la variación del color, la porosidad y el comportamiento hídrico del sustrato pétreo producida por la aplicación del producto consolidante, se realizo su caracterización petrofísica antes y después del proceso de consolidación empleando las siguientes técnicas no destructivas (TND): espectrofotometría, velocidad de propagación de ultrasonidos, determinación de la absorción de agua al vacío y por capilaridad a presión atmosférica. La espectrofotometría se utilizó para medir los parámetros cromáticos sobre la superficie de la parte superior y base de las probetas de piedra antes y después de la consolidación. Para ello, se empleó un espectrofotómetro (MINOLTA CM-700d) considerando los parámetros correspondientes a la escala CIELAB (1976). Se evaluaron los parámetros de luminosidad $\left(\mathrm{L}^{*}\right)$, parámetro rojoverde $\left(a^{*}\right)$, parámetro azul-amarillo $\left(b^{*}\right)$, índices de amarillo (IA) y de blanco (IB) medidos según ASTM 313-76. Se aplicaron las siguientes condiciones de medida: rango de longitud de onda $(\lambda) 400-700 \mathrm{~nm}$, intervalo de $\lambda=10 \mathrm{~nm}$, resolución $0.01 \%$, tiempo de medida $1 \mathrm{seg}$ aprox., modo de medida SCI, rangos de campo de medida de 1 a $3 \mathrm{~mm}$, límite de valores de \pm 1.5 para los parámetros $L^{*} a^{*} b$. Como iluminante estándar se ha seleccionado el D65 con un ángulo de visión o ángulo del observador de $10^{\circ}$. Con los parámetros cromáticos obtenidos, se calculó la variación total de color definida como $\Delta \mathrm{E}^{*}=\left(\left(\Delta \mathrm{L}^{*}\right)^{2}+\left(\Delta \mathrm{a}^{*}\right)^{2}+\left(\Delta \mathrm{b}^{*}\right)^{2}\right)^{1 / 2}$.

La velocidad de propagación de ultrasonidos proporciona información sobre la durabilidad de los materiales y su grado de deterioro. Su magnitud depende del tiempo de propagación del impulso ultrasónico, de la densidad del material y de la presencia de huecos. Se han efectuado medidas directas, consistentes en colocar los transductores (emisor y receptor de ondas ultrasónicas) enfrentados en planos paralelos pero opuestos de las probetas. El aparato de medida utilizado es un equipo portátil PUNDIT C.N.S. Electronics, con transductores de frecuencia $1 \mathrm{MHz}$ y un diámetro de contacto de $10 \mathrm{~mm}$. Para asegurar un buen contacto entre los transductores y la superficie de los materiales, como sustancia acoplante se ha empleado un gel acuoso de carboxymetil celulosa (Sichozell Kleister, Henkel). Se han realizado 12 medidas en cada probeta, en modo directo transmisión/recepción, 4 en cada dirección del espacio, determinando el valor medio, la desviación estándar y la anisotropía total y la relativa. La medida de anisotropía está relacionada con la heterogeneidad de los materiales rocosos y consiste en la medida de la variación de las propiedades de los materiales en función de la dirección en la que se midan. Tanto el índice de anisotropía total $(\mathrm{dM} \%)$ como el relativo $(\mathrm{dm} \%)$, corresponden a las velocidades obtenidas en cada una de las tres direcciones espaciales y ortogonales en cada probeta, teniendo en cuenta la velocidad mínima, la máxima y la intermedia.

La absorción de agua por capilaridad se llevó a cabo para estudiar el coeficiente capilar de las muestras antes y después de la consolidación, mediante un equipo de registro de datos continuo, ACUASOR. El ensayo de absorción de agua bajo vacío se realizó para determinar la densidad real y aparente, la porosidad abierta y para cuantificar la cantidad de agua absorbida por las muestras una vez alcanzada la saturación, según la norma UNE-EN 1936:2007 [16].

\section{RESULTADOS}

\subsection{Caracterización del consolidante}

La solución precursora antes de ser depositada en la cámara a $75 \%$ de humedad relativa fue observada mediante microscopía electrónica de transmisión. La figura 1a muestra una imagen de TEM en campo claro de la solución precursora en la que se identifican cristales hexagonales, no agregados, sin orientación preferencial de hidróxido de calcio, los cuales 


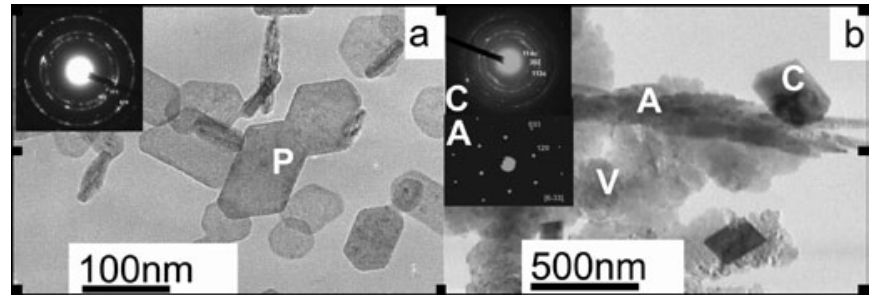

Figura 1. Micrografías en microscopio electrónico de transmisión en campo claro y patrones de difracción de selección de área (saed) del producto consolidante. a) "as deposited" y saed indexado según la portlandita. b) Después de 14 días de exposición al 75\% de humedad relativa, indicando los polimorfos: calcita $(\mathrm{C})$, vaterita $(\mathrm{V})$ y aragonito $(\mathrm{A})$, y patrones saed de la calcita y el aragonito.

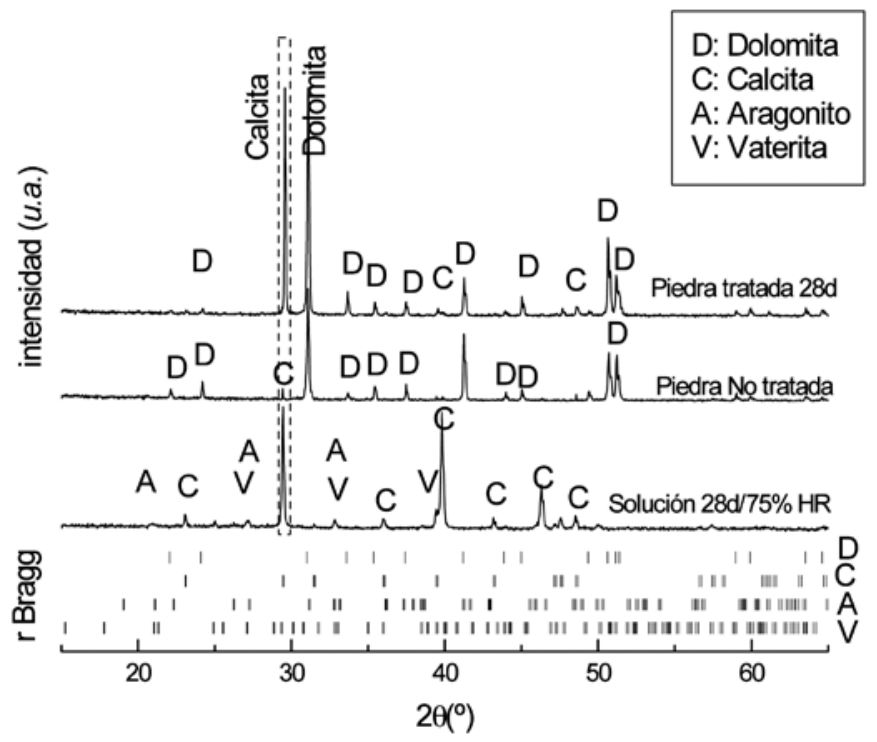

Figura 2. Patrones de difracción de rayos $\mathrm{X}$ de la solución consolidante después de 28 días de exposición al 75\% de humedad relativa; de la piedra no tratada y de la piedra después de 28 días con el tratamiento a una humedad relativa del $75 \%$.

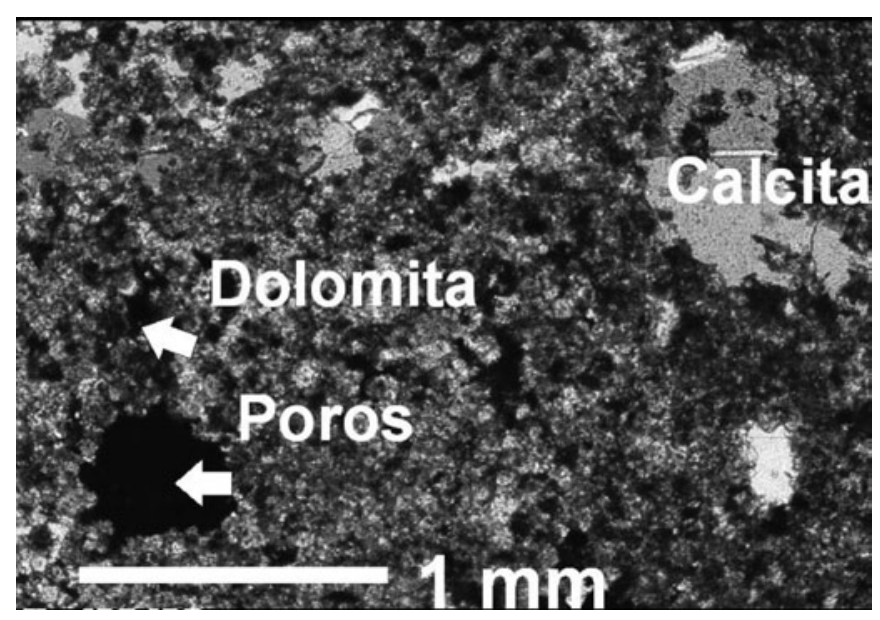

Figura 3 Micrografía en microscopio óptico de luz transmitida (polarizadores cruzados) de la piedra dolomía antes del tratamiento de consolidación. fueron confirmados mediante difracción de electrones como portlandita (JCPDS $=72-0156, \mathrm{P}-3 \mathrm{~m} 1, \mathrm{a}=3,585 \AA, \mathrm{c}=4,895 \AA, \mathrm{SG}=$ 164), con un tamaño de partícula de $103 \pm 30 \mathrm{~nm}$. En la figura $1 \mathrm{~b}$, la imagen TEM en campo claro muestra la misma solución precursora después de 14 días de exposición a 75\% de humedad relativa. En ella se diferencian tres polimorfos de carbonato de calcio correspondientes a cristales romboédricos de calcita (C) con tamaño variable entre 290 y 630nm, cristales fibrosos de aragonito (A) de tamaño entre 1-2,4 $\mu \mathrm{m}$ y cristales aciculares de vaterita $(\mathrm{V})$ con tamaño entre 160 y $260 \mathrm{~nm}$. La identificación de las fases ha sido confirmada mediante difracción de electrones y difracción de rayos X. Estudios realizados sobre la misma solución, después de 21 días de exposición han determinado la presencia de la fase calcita asociada con el carbonato de calcio monohidratado conocido como monohidrocalcita (JCPDS=290306). Los resultados de difracción de rayos $X$ del consolidante después de estar expuesto durante 28 días se observan en la figura 2, donde son comparados con la piedra antes y después de ser tratada con el consolidante. Los máximos de difracción del consolidante coinciden con las reflexiones típicas de la calcita (C), el aragonito (A) y la vaterita (V).

\subsection{Caracterización de la piedra antes y después de la aplicación del consolidante}

\subsubsection{DIFRACCIÓN DE RAYOS X}

En la figura 2 se muestran los patrones de difracción de rayos $\mathrm{X}$ de la piedra antes y después de 28 días del tratamiento con nanopartículas. En la misma figura se indican las posiciones de Bragg de las fases, dolomita (JCPDS=84-1208), calcita (JCPDS=05-0586), aragonito (JCPDS=72-0606) y vaterita (JCPDS=72-1616).

En la piedra no tratada, el patrón de difracción de rayos $X$ muestra los máximos de difracción típicos de la dolomita (20 31, $\left.50,6^{\circ}\right)$, y con menor intensidad máximos correspondientes a la calcita $(2 \theta \sim 29,4)$.

En la piedra tratada, el patrón de difracción de rayos $X$ de la piedra después del tratamiento con nanopartículas indica que después de 28 días, además de las reflexiones típicas de la dolomía (predominantemente fase dolomita), se ha producido un incremento en la intensidad de la fase calcita, siendo bastante notorio en el pico de difracción de mayor intensidad $\left(2 \theta \sim 29,4^{\circ}, h k l=104\right)$.

\subsubsection{CARACTERIZACIÓN MICROSCÓPICA}

La figura 3 muestra la imagen al microscopio óptico de luz transmitida de la dolomía de Redueña antes de su consolidación. Esta roca es una dolomía con estructura masiva constituida mayoritariamente por cristales anhedrales de dolomita $\left(\mathrm{MgCa}\left(\mathrm{CO}_{3}\right)_{2}\right)$. El tamaño de los cristales varía entre $25 \mu \mathrm{m}$ y $93 \mu \mathrm{m}$. En menor proporción se identifican cristales anhedrales de calcita con tamaño mayor a la dolomita llegando a formar cristales de $850 \mu \mathrm{m}$ por procesos de cementación de la porosidad de la roca. La porosidad es fundamentalmente intercristalina (20\%), con morfología regular y tamaño entre 12 y $70 \mu \mathrm{m}$. Igualmente se identifican poros con morfologías más irregulares y de mayor tamaño que generan formas subredondeadas y alargadas, las cuales pueden sufrir coalescencia, con tamaños que alcanzan hasta $1 \mathrm{~mm}$ de diámetro, localmente con relleno de calcita como ha sido previamente descrito. [10], [15]. 
TABLA I. VARIACIONES CROMÁTICAS PRODUCIDAS EN LAS MUESTRAS DE DOLOMÍA DESPUÉS DEL TRATAMIENTO.

\begin{tabular}{llllllll}
\hline Localización & $\boldsymbol{\Delta} \mathbf{L}^{*}$ & $\mathbf{\Delta \mathbf { a } ^ { * }}$ & $\boldsymbol{\Delta} \mathbf{b}^{*}$ & $\boldsymbol{\Delta} \mathbf{C}^{*}$ & $\Delta \mathbf{E}^{*}$ & $\Delta \mathbf{I A}^{*}$ & $\Delta \mathbf{I B}^{*}$ \\
\hline Parte superior & $-0,12$ & $-1,04$ & $-2,03$ & 2,28 & 2,28 & $-3,78$ & $-7,41$ \\
Parte inferior & $-1,86$ & 0,6 & 2,08 & 2,85 & 2,85 & 3,55 & $-9,5$ \\
\hline
\end{tabular}

La piedra, al microscopio electrónico de barrido, (Figuras 4a-4c) muestra predominantemente cristales romboédricos de dolomita $(40-55 \mu \mathrm{m})$ aunque localmente pueden llegar a tamaños hasta $100 \mu \mathrm{m}$, a menudo desarrollando caras cóncavas y agregados cristalino-granulares con superficies altamente porosas (entre $5-21 \mu \mathrm{m})$. Se identifican cristales predominantemente tabulares de calcita esparítica que llegan a tener un tamaño de $440 \mu \mathrm{m}$, generados por un proceso de de-dolomitización de la matriz dolomítica desarrollando localmente romboedros (Fig. 4a y 4c). Hay un predominio de porosidad intra-cristalina que afecta predominantemente a la dolomita, sobre la porosidad inter-cristalina, más común en los contactos entre planos calcíticos-dolomíticos. Los espacios porales se dividen en poblaciones de poros pequeños, entre $6 \mathrm{y}$ $12 \mu \mathrm{m}$, que son comunes en el contacto calcita-dolomita, poros medianos entre $45 \mu \mathrm{m}$ y $70 \mu \mathrm{m}$ y poros grandes variables entre 100 a $500 \mu \mathrm{m}$, que afectan a la matriz dolomítica.

Tal y como ha sido observado mediante las imágenes de ESEM, obtenidas sobre la cara superior de la probeta, el consolidante aplicado a la piedra después de 28 días de la aplicación del consolidante y expuesta a $75 \%$ de humedad relativa (Figura $4 \mathrm{~b}$ ) ha penetrado en los planos de clivaje asociados a la calcita y en los espacios porosos entre la calcita y la dolomita. Un detalle de la carbonatación, se aprecia en el suavizado de la superficie que se aprecia en la imagen amplificada de la piedra después de la consolidación (figura 4d) en comparación con la misma antes de consolidar (figura 4c). La figura 5 muestra un detalle de las figuras 4 c y $4 d$, en donde se aprecia la porosidad de la piedra antes (figura 5a) y después de 28 días de tratamiento (figura $5 b$ ). Sobre esta zona se ha realizado un estudio estadístico de la porosidad basado en el análisis de las imágenes de microscopía electrónica de barrido, comparando los diámetros de poros. Los resultados obtenidos se han graficado en los histogramas de frecuencias de la distribución del tamaño de poro antes (figura 5c) y después de aplicar el producto (figura 5d). A partir de las medidas se deduce que el consolidante no rellena los poros mayores de $110 \mu \mathrm{m}$ de diámetro; sin embargo, ha rellenado los poros predominantemente en el intervalo entre 30 y $110 \mu \mathrm{m}$ de diámetro. En algunos casos se ha producido el relleno total de estos poros, mientras que en otros, se ha producido un relleno parcial que ha dado lugar a un ligero incremento de los poros de tamaño menor de $10 \mu \mathrm{m}$.

\subsubsection{CARACTERIZACIÓN PETROFÍSICA}

Los resultados de espectrofotometría se resumen en la Tabla I, indicando los parámetros cromáticos de la piedra antes de ser tratada y después de aplicar el consolidante. Se aprecia un ligero cambio cromático con tendencia al oscurecimiento de la superficie tratada al disminuir el índice de Luminosidad $\left(L^{*}\right)$ en 1,86 unidades y un incremento de la Croma $\left(C^{*}\right)$ de 2,85 unidades. El parámetro cromático que más cambia, al tratar a la dolomía con el consolidante, es el índice de Blanco (IB) que disminuye en 8,56 unidades. El índice de amarillo tiende a incrementarse, (2,51 unidades). Las modificaciones de color
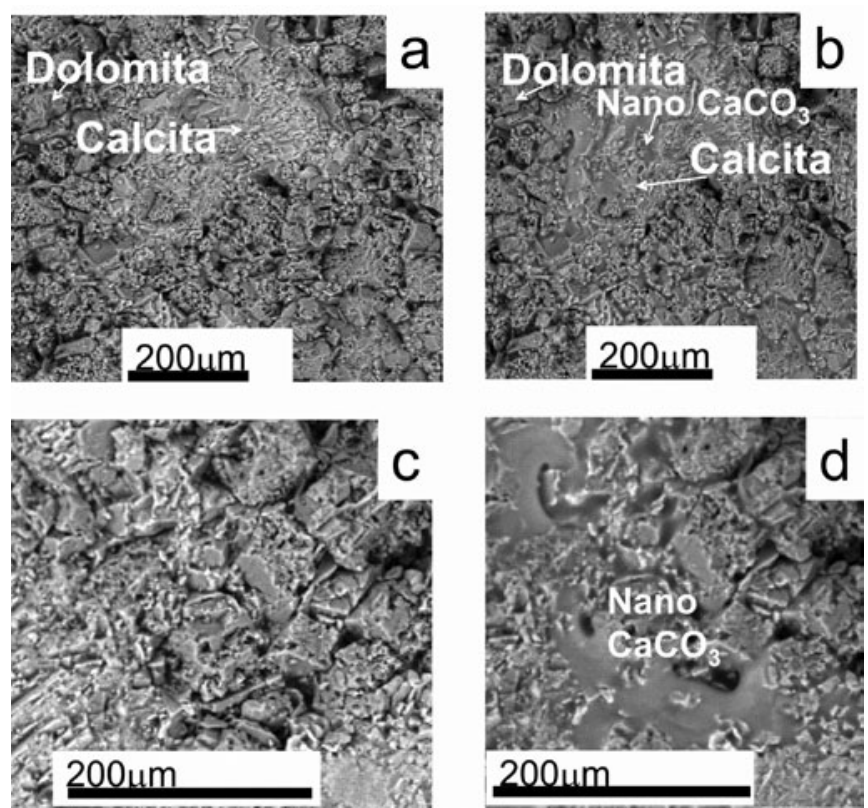

Figura 4. Micrografías en microscopio electrónico de barrido en modo de operación ambiental (ESEM) de la piedra antes del tratamiento (Figuras 4a y 4c) y después de 28 días de aplicación del consolidante (Figuras $4 \mathrm{~b}$ y $4 \mathrm{~d}$ ). Cara superior de la probeta.
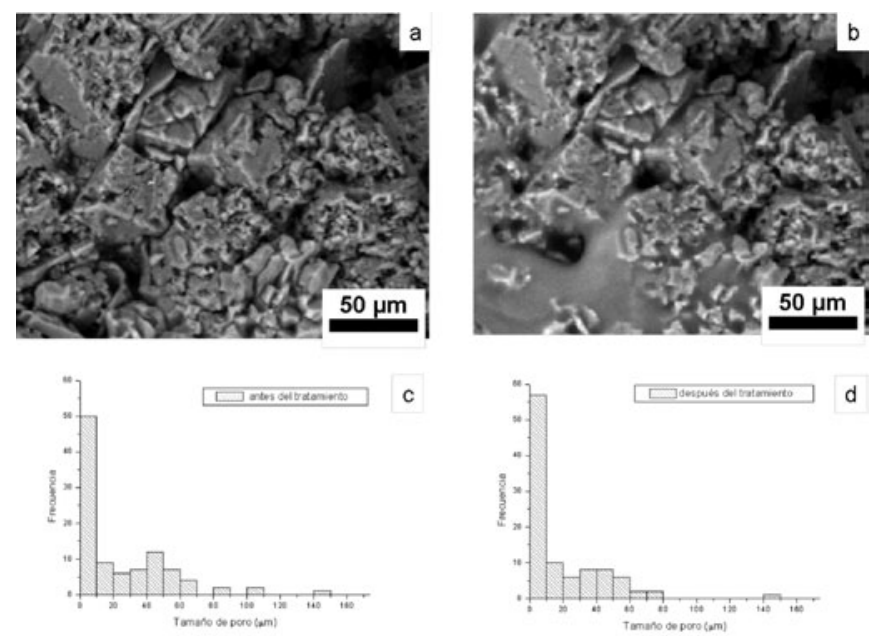

Figura 5 Detalle de las figuras 4c y 4d sobre la cara superior de la probeta mostrando la porosidad de la piedra antes $(5 a)$ y después de 28 días de tratamiento (5b). Histogramas de frecuencias de la distribución del tamaño de poro antes (5c) y después del tratamiento (5d), obtenido a partir de las medidas realizadas en la región seleccionada. 
que genera el tratamiento se pueden considerar que no alteran la percepción cromática de la superficie tratada al tener un cambio global del color $\left(\Delta \mathrm{E}^{*}=2,85\right)$ inferior a 5 unidades.

Los datos obtenidos de la caracterización hídrica de las muestras antes y después de la consolidación se resumen en la Tabla II. Los resultados del ensayo de absorción de agua a vacío indican que se produce un muy ligero descenso de la porosidad abierta y de la saturación de agua después de la consolidación de las muestras. Sin embargo, los resultados del ensayo de absorción de agua por capilaridad indican que las muestras sufren un descenso del coeficiente de capilaridad (C) del 33\% aproximadamente.

Los resultados obtenidos de las medidas de propagación de velocidad de ultrasonidos se resumen en la Tabla III Se observa que como resultado de la consolidación hay un aumento en la velocidad media de la transmisión ultrasónica a través del material en cerca de un $12 \%$. A partir de los valores de velocidad de ultrasonidos, antes de la consolidación, se detecta un grado de anisotropía muy bajo, tanto del índice de anisotropía absoluto (dM) como del relativo (dm). Al consolidar, la dolomía aumenta notablemente su anisotropía, duplicando el valor de la anisotropía absoluta, y multiplicando por 10 el valor de la anisotropía relativa.

\section{DISCUSIÓN DE RESULTADOS}

El proceso de carbonatación ha sido confirmado tanto en la transformación de fase a partir de portlandita en el producto utilizado como consolidante, como en el efecto del mismo sobre el substrato pétreo al cual fue aplicado. La transformación de fase en las nanopartículas ha sido completa generando los polimorfos de carbonato de calcio, calcita, aragonito y vaterita, sin evidencias de la fase precursora portlandita incluso a los 14 días de exposición. No obstante, dicha solución al finalizar los 28 días de exposición a la humedad relativa del $75 \%$ es heterogénea estructuralmente. La existencia de las tres fases anhidras polimórficas está indicando que aún no se ha conseguido la estabilidad estructural. Evidencia de ello es la presencia de las fases inestables aragonito (ortorrómbico) y vaterita (hexagonal), con un crecimiento cristalino distinto, que puede llegar a afectar los resultados del comportamiento hídrico una vez aplicados en la piedra. Un comportamiento similar en cuanto al proceso de transformación de fases ha sido obtenido utilizando una solución coloidal de nanorestore ${ }^{\circledR}$ con mayor concentración [11], aunque en este caso el grado de cristalinidad es más bajo identificándose alto contenido de carbonato de calcio amorfo y monohidrocalcita. Los resultados obtenidos aquí, son comparables con los ensayos realizados a $90 \%$ de humedad relativa en iguales condiciones utilizando el mismo producto consolidante. En esas condiciones, el proceso de formación de la calcita es más rápido y a 28 días de tratamiento las fases inestables han sido transformadas totalmente a calcita [17].

En el proceso de carbonatación, el $\mathrm{CO}_{2}$ presente en el ambiente reacciona con el $\mathrm{Ca}(\mathrm{OH})_{2}$ en presencia de una humedad relativa alta generando el $\mathrm{CaCO}_{3}$, siendo la principal causa de endurecimiento en diferentes materiales. Sin embargo, factores como la concentración de $\mathrm{CO}_{2}$ en la atmósfera o el contenido de agua como fase vapor influyen en el proceso de saturación. Es por esto que la difusión del $\mathrm{CO}_{2}$ a altas humedades relativas puede estar condicionada por la presencia de la fase vapor, o por iones hidroxilo que pueden estar presentes dentro de los poros. El proceso de difusión del anhídrido carbónico solo es posible cuando los poros se encuentran llenos de aire, lo que realmente está controlando el proceso de carbonatación en los espacios porales.

Las pequeñas diferencias manifestadas principalmente a partir de las medidas de ultrasonidos y anisotropía están relacionadas con el avance del consolidante, quien va rellenando los poros de acuerdo a su geometría, llegando a generar una anisotropía paralela al avance del ascenso capilar. El carbonato de calcio puede llegar a colmatar los poros de la dolomía al formar una superficie, lo que puede llegar a cerrar algunos de ellos e impedir el avance de la reacción. Este comportamiento está directamente relacionado con la manera como ha sido aplicado el producto. La penetración del tratamiento alcanza los 1,5 centímetros de espesor de la probeta, debido a que la aplicación realizada por capilaridad ha ascendido a lo largo de toda la probeta, hasta colmatar la superficie superior de la misma, tal como se aprecia en la Figura 5.

De hecho, el incremento en la anisotropía, está muy relacionado con el método de consolidación, por absorción capilar de la solución con las nanopartículas, que ha debido generar una especie de corteza o franja consolidada mediante los frentes de ascensión capilar, principal responsable del aumento de velocidad en el eje $\mathrm{z}$, y por tanto del aumento de la anisotropía absoluta o total, en la que se ven implicadas las medidas tomadas en los tres ejes $\mathrm{x}, \mathrm{y}, \mathrm{z}$. La anisotropía relativa, que es la que más aumenta, es la que se concentra en el plano xy. Es bastante claro que la distribución del consolidante en este plano debe obedecer a la estructura cristalina del material, y por lo que se observa a partir de las observaciones de ESEM y DRX, es la calcita la que inicialmente imprime una orientación más marcada al material, ya que es a través de la red porosa que dejan los cristales de calcita por donde se observa que el consolidante rellena y reduce porosidad, lo cual no se observa tanto en las zonas de cristales dolomíticos, que parecen dejar poros más grandes, y que la solución de nanopartículas no aprovecha de igual modo los capilares más estrechos que genera la calcita. Así es que por todo ello, se considera que la consolidación sigue la orientación inicial de la estructura calcítica inicial, y que la marca aún más, sin descartar

TABLA II. RESULTADOS DE LOS ENSAYOS DE CARACTERIZACIÓN HÍDRICA: ABSORCIÓN DE AGUA, POR CAPILARIDAD A PRESIÓN ATMOSFÉRICA Y AL VACÍO, ANTES Y DESPUÉS DE LA CONSOLIDACIÓN DE LAS MUESTRAS.

\begin{tabular}{|c|c|c|c|c|c|c|c|c|}
\hline \multicolumn{2}{|c|}{ Coeficiente capilar (C) $\left(\mathrm{g} / \mathrm{m}^{2} \cdot \mathrm{s}^{0.5}\right)$} & \multirow{2}{*}{$\begin{array}{l}\Delta \mathrm{C}(\%) \\
-33,82\end{array}$} & \multicolumn{2}{|c|}{ Saturación (S) (\%) } & \multirow{2}{*}{$\begin{array}{c}\Delta \mathbf{S}(\%) \\
-0,05\end{array}$} & \multicolumn{2}{|c|}{ Porosidad abierta (P) (\%) } & \multirow{2}{*}{$\begin{array}{r}\Delta \mathbf{P}(\%) \\
-0,04\end{array}$} \\
\hline Antes & Después & & Antes & Después & & Antes & Después & \\
\hline 143,66 & 107,35 & & 10,14 & 10,09 & & 22,31 & 22,27 & \\
\hline
\end{tabular}


procesos de fisuración orientada asociada a la consolidación, fenómenos de disolución localizada, o de efectos cuña. Carlson 1983 [18], indica que la nucleación de la calcita, dentro de los espacios porosos, suele ocurrir de manera heterogénea en forma de crecimientos orientados epitaxiales; indica además que la morfología y orientación de los cristales de calcita van a estar controladas por el aragonito precursor. En el caso de estudio, se ha comprobado mediante DRX sobre la muestra del consolidante, que puede llegar a existir a los 28 días en muy baja proporción la fase aragonito asociado a calcita. Es por tanto probable que aunque la presencia de la fase inestable aragonito en el substrato pétreo analizado por el mismo método puede estar en muy baja proporción, por debajo del límite de detección de la difracción de rayos $\mathrm{X}$, contribuya a la modificación del comportamiento anisotrópico de la piedra tratada. Incluso, no se puede descartar el efecto de la presencia en pequeñas proporciones de la fase vaterita, la más inestable de todos los polimorfos de carbonato de calcio.

Dado que el proceso de precipitación de los carbonatos de calcio se realiza en condiciones especiales a partir de nanocristales de portlandita en una solución coloidal alcohólica, se deben tener en cuenta diferentes variables como son el posible intercambio iónico $\mathrm{Mg}^{2+} / \mathrm{Ca}^{2+}$ que puede existir en el entorno piedra-consolidante, la presencia de moléculas de agua o grupos hidroxilo que pueden entrar en la estructura cristalina de los carbonatos tanto de calcio como de magnesio, el efecto del tamaño sobre la cinética de carbonatación y el efecto de la diferencia en la tensión superficial en el medio. En el proceso, además de los típicos polimorfos anhidros pueden llegar a precipitarse fases inestables hidratadas como la monohidrocalcita e incluso puede darse el caso de formar soluciones sólidas con diferentes relaciones $\mathrm{Mg} / \mathrm{Ca}$ en donde pueden llegar o no a estar implícitas moléculas de agua. La transformación de fase a partir de estas soluciones sólidas puede generar modificaciones en la energía libre de formación, lo que se refleja en modificaciones locales en el volumen de los polimorfos y que actúan modificando localmente la estructura porosa.

$\mathrm{El}$ intercambio $\mathrm{Mg}^{2+} \mathrm{Ca}^{2+}$ en rocas dolomíticas en ambientes acuosos ha sido ampliamente estudiado [19], y se ha aplicado a condiciones específicas como soluciones acuosas y estabilidad en poros y microporos en ambientes marinos, donde se ha determinado el papel fundamental que ejerce la relación $\mathrm{Mg}^{2+}$ / $\mathrm{Ca}^{2+}$ sobre la precipitación de las fases de carbonatos.

Este efecto de la carbonatación ha sido valorado en nanopartículas suspendidas en isopropanol con diferente concentración obtenidas por otros métodos de síntesis [10], [20] . En pruebas llevadas a cabo por Daniele y Taglirei 2010 [20], al 70\% de humedad relativa, el agua residual y la concentración afectan significativamente la eficiencia de la carbonatación. Los ensayos realizados por López-Arce et al 2010 [10] sobre dolomías utilizando como consolidante nanopartículas de hidróxido de calcio en solución isopropílica con una concentración de $5 \mathrm{~g} / 1$ (nanorestore ${ }^{\circledR}$ ), han conseguido rellenar los poros y los contactos entre granos en la dolomita. Sin embargo, a diferencia de los resultados obtenidos en el presente trabajo, dicha concentración no ha favorecido la recristalización de la calcita en condiciones de alta humedad relativa, produciendo un incremento en la velocidad de ultrasonidos, una mayor disminución en la porosidad abierta y en los valores de saturación, así como un descenso en el coeficiente capilar de las muestras tratadas. Este descenso implica una menor velocidad de transporte de agua a través de los poros capilares, y por tanto una mayor durabilidad de la piedra a largo plazo [21]. Esto se puede explicar por una mejora en la cohesión de las muestras tratadas, en las que se favorece la reducción de porosidad inter-cristalina, dando lugar a una menor porosidad conectada, una velocidad menor de absorción capilar de agua y por tanto menor coeficiente de absorción capilar. El método de absorción continua aplicado permite una caracterización más precisa de los materiales de construcción con altas velocidades de absorción $(C>10 \mathrm{~kg} /$ $\mathrm{m}^{2} \mathrm{~h}^{0.5}$ ) y con áreas de contacto estrechas en comparación con el método tradicional no continuo [22]. Cueto et al, 2009 [21] determinan que además de las propiedades físicas de la piedra, como la porosidad, la capilaridad también está controlada por la mineralogía del cemento-matriz, tamaño de clastos y distribución espacial de todos los elementos texturales dentro de la roca. Estos autores establecen que existe una relación directa entre cemento dolomítico y el coeficiente de capilaridad. Por el contrario, un alto contenido en cemento calcítico facilita

TABlA III. RESUltAdOS DE LA MEDIDA DE LA VELOCIDAD DE TRANSMISIÓN ULTRASÓNICA Y DE LOS ÍNDICES DE ANISOTROPÍA ANTES (A) Y DESPUÉS (D) DE LA CONSOLIDACIÓN DE LAS PROBETAS.

\begin{tabular}{|c|c|c|c|c|c|c|}
\hline \multirow[b]{2}{*}{ Eje } & \multicolumn{4}{|c|}{ Velocidad de ultrasonidos } & \multicolumn{2}{|c|}{ Anisotropía } \\
\hline & & $\mathrm{m} / \mathrm{s}$ & $\begin{array}{l}\text { Valor medio } \\
(\mathrm{m} / \mathrm{s})\end{array}$ & $\begin{array}{l}\Delta \mathrm{Vp} \\
(\%)\end{array}$ & dM & $\mathrm{dm}$ \\
\hline \multirow{3}{*}{$x$} & Antes & $2540 \pm 25$ & \multirow{9}{*}{$\begin{array}{l}\text { A: } 2571 \pm 70 \\
\text { D: } 2872 \pm 73\end{array}$} & \multirow{9}{*}{$+11,7 \%$} & \multirow{9}{*}{$\begin{array}{l}\text { A:1,76 } \\
D: 3,71\end{array}$} & \multirow{9}{*}{$\begin{array}{l}\text { A: } 0,34 \\
\text { D: } 3,67\end{array}$} \\
\hline & Después & $2801 \pm 32$ & & & & \\
\hline & $\Delta \mathbf{V} \mathbf{p}_{\mathrm{x}}$ & $+10,3 \%$ & & & & \\
\hline \multirow{3}{*}{$\mathbf{Y}$} & \multirow{3}{*}{$\begin{array}{c}\text { Antes } \\
\text { Después } \\
\Delta V \mathbf{p}_{\mathrm{y}} \\
\end{array}$} & \multirow{3}{*}{$\begin{array}{c}2590 \pm 51 \\
2855 \pm 59 \\
+10,2 \%\end{array}$} & & & & \\
\hline & & & & & & \\
\hline & & & & & & \\
\hline \multirow{3}{*}{$\mathrm{Z}$} & Antes & $2581 \pm 135$ & & & & \\
\hline & Después & $2962 \pm 127$ & & & & \\
\hline & $\Delta \mathbf{V} \mathbf{p}_{\mathrm{z}}$ & $+14,8 \%$ & & & & \\
\hline
\end{tabular}


la reducción de la absorción capilar, lo cual explica en parte la reducción del coeficiente capilar de las muestras tratadas con nanopartículas de $\mathrm{Ca}(\mathrm{OH})_{2}$ en las que al ser expuestas a condiciones de alta HR se produce la carbonatación de las mismas dando lugar a una cementación calcítica, aumentando la proporción de calcita en los poros capilares de las muestras.

Respecto a las variaciones en los parámetros cromáticos después del tratamiento, tan sólo se producen pequeñas modificaciones en la parte inferior de las probetas (la parte que ha estado en contacto directo con el consolidante) dónde se produce un ligero amarillamiento como muestran los valores positivos de $\Delta \mathrm{IA}^{*}$. Los valores negativos tanto de la luminosidad $\left(\Delta \mathrm{L}^{*}\right)$ como de la variación del índice de blancos $\left(\Delta \mathrm{IB}^{\star}\right)$ pueden interpretarse como un ligero oscurecimiento en las probetas tratadas mostrando que la intensidad del color de los materiales pétreos decrece ligeramente con la aplicación del producto consolidante, siendo esta variación mayor en las zonas con mayor contacto con el consolidante. Tanto la parte superior como la inferior muestran una diferencia de color $\left(\Delta \mathrm{E}^{*}\right)$ menor de 3 , por lo que el producto consolidante no afecta significantemente a los parámetros colorimétricos de los materiales pétreos después del tratamiento. Esta diferencia es menor de 5 según Normal 20/85, 1996 [23], lo que implica que al tener una diferencia menor de 3 se encuentre por debajo del umbral de la percepción y no puede ser detectada visualmente [24], [25], [26].

\section{CONCLUSIONES}

La utilización de nanopartículas de portlandita en una solución coloidal isopropílica para la consolidación de piedra natural de naturaleza carbonática se ha mostrado eficaz al conseguir un aumento del grado de cohesión de la roca con un incremento de la velocidad de propagación de ultrasonidos. La transformación del hidróxido de calcio a fases carbonatadas de calcio se obtiene en condiciones de humedad relativa altas, en donde las fases más inestables aragonito y vaterita van pasando a fases más estables con cristales de calcita con el paso del tiempo necesitando al menos 28 días para la transformación total a calcita. La consolidación de la dolomía estudiada se realiza siguiendo la estructura y orientación de las fases cristalinas primarias realizándose la nucleación de la calcita dentro del sistema poroso de la roca y siguiendo a su vez la orientación de los cristales de aragonito precursores del proceso de transformación. Esto da lugar a un aumento de la anisotropía, ocasionada por el frente de penetración del tratamiento en el interior de la roca y a la orientación de la calcita durante dicho proceso de transformación dentro del sistema poroso de la roca. También se genera una disminución importante de la absorción de agua capilar lo que hace que este tratamiento, además de consolidar la roca, permita mejorar su resistencia a los procesos de deterioro por acción hídrica.

La aplicación de este tratamiento no ha generado alteraciones significativas de las propiedades cromáticas del sustrato, ni la presencia de subproductos que pudieran generar daños en el material, por lo que también se puede indicar que este tratamiento es idóneo para mejorar la calidad de la piedra tratada teniendo como ventaja sobre otros tratamientos basados en productos poliméricos, el ser compatible con la naturaleza química de la piedra ya que es un medio natural.

\section{AGRADECIMIENTOS}

A los programas Geomateriales (S2009/MAT-1629) y CONSOLIDER-TCP (CSD2007-0058) y a la financiación de grupo de investigación de la UCM: Alteración y Conservación de los materiales pétreos del Patrimonio (ref. 921349). Igualmente al Programa JAE del CSIC. A la ICTS-Centro Nacional de Microscopía Electrónica, al CAI de rayos X de la UCM y al Museo de Ciencias Naturales (CSIC).

\section{REFERENCIAS}

1. R. Fort. "Polímeros sintéticos para la conservación de materiales pétreos". en: Ciencia, Tecnología y Sociedad para una conservación sostenible del patrimonio pétreo. (2007). Pérez-Monserrat, E.M., Gómez-Heras, M. Álvarez de Buergo, M., Fort, R. (eds). Dpto. Publicaciones Universidad Popular José Hierro, San Sebastián de los Reyes: 71-82. ISBN: 84-95710-41-2

2. R. Giorgi, M. Baglioni, D. Berti, P. Baglioni. (2010) "New methodologies for the conservation of Cultural Heritage: Micellar solutions, microemulsions and hydroxide nanoparticles". Accounts of Chemicals research, 695-704 (2010)

3. P. Baglioni, R. Giorgi. "Soft and hard nanomaterials for restoration and conservation of cultural heritage". Soft. Matter, 293-303 (2006).

4. AH. De Aza, MA. Rodriguez JL. Rodriguez, S. De Aza P. Pena. “Decomposition of Dolomite Monitored by Neutron Thermodiffractometry", Journal Of The American Ceramic Society 85, 881-888 (2002).

5. M. Alvarez de Buergo, R. Fort, M. Gomez-Heras. "Contributions of SEM to the assessment of the effectiveness of stone conservation treatments". Scanning 26[1] 41-47 (2004)

6. J. Ashurst, F.G. Dimes. "The cleaning and treatment of limestone by the lime method". Conservation of Building and Decorative Stone 2, 169-84 (1990).

7. C. Rodriguez-Navarro, E. Ruiz-Agudo, M. Ortega-Huertas, E. Hansen. “Nanostructure and irreversible colloidal behavior of $\mathrm{Ca}(\mathrm{OH}) 2$ : Implications in cultural heritage conservation". Langmuir 21, 10948-10957 (2005).

8. C. Price, K. Ross, G. White. "A further appraisal of the 'Lime Technique' for limestone consolidation, using a radioactive tracer". Studies in Conservation 33, 178-186 (1988).

9. V. Daniele, G. Taglieri, R. Quaresima. "The nanolimes in Cultural Heritage conservation: Characterisation and analysis of the carbonation process". J. Cult. Herit., 9, 3, 294-301 (2008)

10. P. Lopez-Arce, LS. Gomez-Villalba, L. Pinho, M. E. Fernández-Valle, M. Álvarez de Buergo, R. Fort.,2010. "Influence of porosity and relative humidity on consolidation of dolostone with calcium hydroxide nanoparticles: Effectiveness assessment with non-destructive techniques". Mat. Character. 61[2] 168-184 (2010).

11. P. López-Arce, L.S. Gómez-Villalba, S. Martínez-Ramírez, M. Álvarez de Buergo R. Fort. "Influence of relative humidity on the carbonation of calcium hydroxide nanoparticles and the formation of calcium carbonate polymorphs". Powder Technol., 205, 263-269 (2011)

12. L.S. Gómez -Villalba, P. López-Arce, R. Fort González, M Álvarez de Buergo, “La aportación de la nanociencia a la conservación de bienes del patrimonio cultural". Patrimonio Cultural de España", v. 4, 43-56, 2010.

13. E. Hansen, E. Doehne, J. Fidler, J. Larson, B. Martin, M. Matteini, C. RodriguezNavarro, E. Sebastián Pardo, C. Price, A. de Tagle, J.M. Teutonico, N. Weiss N. “A review of selected inorganic consolidants and protective treatments for porous calcareous materials". Reviews in Conservation 4, 13-25 (2004).

14. G. Ziegenbald. "Colloidal calcium hydroxide- a new material for consolidation and conservation of carbonate stone". Proceedings of 11th International congress on deterioration and conservation of stone III: 1109. (2008)

15. R. Fort, B. Fernández-Revuelta, M.J. Varas, , M. Álvarez de Buergo, M. Taborda, "Effect of anisotropy on Madrid-region Cretaceous dolostone durability in salt crystallization processes" Materiales. Construccion., 58, 289-290, 161-177.(2008).

16. UNE-EN 1936:2007 "Métodos de ensayo para piedra natural. Determinación de la densidad real y aparente y de la porosidad abierta y total" (207)

17. L. S. Gómez-Villalba, P. Lopez-Arce, M. Alvarez de Buergo, R. Fort. “Comportamiento cristalino de Nanopartículas de Portlandita $\left(\mathrm{Ca}(\mathrm{OH})_{2}\right)$ en condiciones de alta humedad relativa“. Macla, 13, 103-104 (2010)

18. W. D. Carlson. "Polymorphs of calcium carbonate and the aragonite-calcite transformation". Rev. Mineral., 11,191-225 (1983)

19. F.T. Mackenzie, W.D. Bischoff, F.C. Bishop, M. Loijens, J. Schoonmaker, R. Wollast. "Magnesian calcites: low temperatura occurence, solubility and solid -solution behaviour". Rev. Mineral., 11, 97-144 (1983)

20. V. Daniele, G. Taglieri. "Nanolime suspensions applied on natural lithotypes: The influence of concentration and residual water content on carbonatation process and on treatment effectiveness". J. Cult. Herit., 11, 102-106 (2010)

21. N. Cueto, D. Benavente, J. Martínez-Martínez, M. A. García-del-Cura. “Rock fabric, pore geometry and mineralogy effects on water transport in fractured fabric, pore geometry and mineralogy effect
dolostones“. Eng. Geol., 107, 1-2, 1-15(2009)

22. D. Benavente, N. Cueto, J. Martinez-Martinez, M. A. García del Cura, J.C. Cañaveras. "The role of petrophysical properties of porous building rocks on salt weathering". Environ. Geol., 52, 197-206 (2007)

23. NORMAL 20/85. "Interventi conservativi: progettazione esecuzione e valutazione preventive“", Milan, Italy (1996)

24. C. M. Grossi, P. Brimblecombe, R. M. Esbert, F. J. Alonso. “Color Changes in Architectural Limestones from Pollution and Clearing". Color Res. Appl., 32, 4, 320-331 (2007)

25. D. Benavente, F. Martínez-Verdú, A. Bernabeu, V. Vigueira, R. Fort, M. A. García del Cura, C. Ilueca, S. Ordoñez. "Influence of surface roughness on color changes in building stones". Color Res. Appl., 28, 343-351 (2003)

26. J. Delgado Rodrigues, A. Grossi. "Indicators and rating for the compatibility asessment of conservation actions". J. Cult. Herit., 8, 32-43 (2007).

Recibido: $15 / 11 / 2010$

Aceptado: 01/04/2011 